\title{
Epidemiology, etiology, and pathogenesis of tendinopathy among athletes
}

\author{
Syed Asif Hasan ${ }^{1 *}$, Ahmed Abdulrahman Othman², Yousuf Kamal Shagdar ${ }^{3}$, \\ Abdulrahman Anis Khan ${ }^{4}$, Amal Baalqasim Sulaimani ${ }^{5}$, Hassan Ali Alsinan ${ }^{6}$, \\ Ahmed Abdullah Almenhali ${ }^{7}$, Wael Abdulrahman Al Luhayb ${ }^{8}$, \\ Ahmed Abdulrahman Alrashidi ${ }^{9}$, Muath Abdulaziz Alghareeb ${ }^{10}$, Raad Saad Aldakheel ${ }^{8}$
}

\author{
${ }^{1}$ Department of Orthopaedics, Sameera Medical Center, Jeddah, Saudi Arabia \\ ${ }^{2}$ College of Medicine, Lugansk State Medical University, Luhansk, Ukraine \\ ${ }^{3}$ College of Medicine, King Abdulaziz University in Rabigh, Rabigh, Saudi Arabia \\ ${ }^{4}$ Department of Orthopaedics, King Abdulaziz Hospital, Jeddah, Saudi Arabia \\ ${ }^{5}$ Department of Orthopaedics, King Salman Medical City, Medina, Saudi Arabia \\ ${ }^{6}$ College of Medicine, Ibn Sina National College, Jeddah, Saudi Arabia \\ ${ }^{7}$ College of Medicine, King Abdulaziz University, Jeddah, Saudi Arabia \\ ${ }^{8}$ College of Medicine, King Khalid University, Abha, Saudi Arabia \\ ${ }^{9}$ College of medicine, Qassim university, Qassim, Saudi Arabia \\ ${ }^{10}$ College of Medicine, Imam Mohammad Ibn Saud Islamic University, Riyadh, Saudi Arabia
}

Received: 20 December 2021

Accepted: 03 January 2021

\section{*Correspondence:}

Dr. Syed Asif Hasan,

E-mail: drasifh@gmail.com

Copyright: ( ) the author(s), publisher and licensee Medip Academy. This is an open-access article distributed under the terms of the Creative Commons Attribution Non-Commercial License, which permits unrestricted non-commercial use, distribution, and reproduction in any medium, provided the original work is properly cited.

\section{ABSTRACT}

The treatment of tendinopathy can be challenging for clinicians and might be associated with serious adverse events. However, evidence shows that tendinopathy can significantly impact the professional careers of the affected athletes. The main clinical features of the condition include decreased performance and strength of the affected tendons, swelling, and localized pain. Although tendinopathy might seem a non-critical condition, estimates show that it might be careerending for many professional athletes, and therefore, reducing their quality of life. In the present literature review, we have discussed the epidemiology, etiology, and pathogenesis of tendinopathy among athletes. Different intrinsic and extrinsic factors were found to contribute to the development of the condition and the impact of various systemic diseases. Evidence also shows that the prevalence of the condition is high among athletes. However, these rates were variable across the different studies in the literature. This is probably owing to various factors, like demographics and the process of detection. The pathogenesis of the condition is also very complicated, and the development of pain has been attributed to the process of angiogenesis and associated ingrowth of nerve fibers.

Keywords: Tendinopathy, Tendon lesion, Physiotherapy, Pathophysiology, Epidemiology, Etiology

\section{INTRODUCTION}

Tendinopathy is a common disorder that affects athletes and non-athletes. The condition is not critical. However, it can be disabling and reduce daily activities, impacting the quality of life. Patellar and Achilles tendons are the most commonly affected in the lower limbs. On the other hand, extensor carpi radialis brevis and rotator cuff are the most frequently affected tendons in the lower limbs. ${ }^{1}$

The treatment of tendinopathy can be challenging for clinicians and might be associated with serious adverse events. However, evidence shows that tendinopathy can significantly impact the professional careers of the affected 
athletes. The main clinical features of the condition include decreased performance and strength of the affected tendons, swelling, and localized pain. ${ }^{2}$ In addition, although it has been frequently reported that the condition is usually associated with increasing age, a previous report showed that different age groups with variable physical activities could be impacted by retinopathy. ${ }^{3}$ Finally, recovery from the condition is slow, and evidence indicates that the treatment is challenging. ${ }^{4}$

Studying the epidemiology, etiology, and pathogenesis of the condition might help clinicians and researchers to understand the impact of the disease properly. This can urge them to apply more adequate interventions and innovative approaches to reduce exposure to the different risk factors. ${ }^{3,5}$ Consequently, we aim to conduct this literature review to discuss the epidemiology, etiology, and pathogenesis of tendinopathy in athletes based on evidence from studies in the literature.

\section{METHODS}

This literature review is based on an extensive literature search in Medline, Cochrane, and EMBASE databases which was performed on $27^{\text {th }}$ November 2021 using the Medical subject headings (MeSH) or a combination of all possible related terms, according to the database. To avoid missing potential studies, a further manual search for papers was done through Google Scholar while the reference lists of the initially included papers. Papers discussing epidemiology, etiology, and pathogenesis of tendinopathy among athletes were screened for useful information. No limitations were posed on date, language, age of participants, or publication type.

\section{DISCUSSION}

\section{Epidemiology}

Estimates show that around $50 \%$ of athletes suffer from tendon injuries. This rate has been reportedly increasing due to the increasing rate of sporting activities worldwide. ${ }^{3}$ In addition, evidence shows that chronic overuse has been associated with the development and pathogenesis of tendinopathy. For instance, a previous study estimated that an adjusted odds ratio of 31.2 for Olympic runners was associated with increased incidence of Achilles tendinopathy compared with their age-matched controls. ${ }^{6}$ In addition, an earlier report that included elite soccer players also demonstrated that the risk of patellar tendinopathy was associated with the total hours spent with sports practicing activities. ${ }^{7}$ The risk of developing tendinopathy has also been associated with prolonged loading over tendons and frequency of practicing tiring activities without adequate rest intervals. ${ }^{8}$

Epidemiological studies reported various prevalence rates of the condition with multiple risk factors. Estimates indicate the Achilles tendinopathy among athletes is one of the most commonly encountered lower limb overuse- related injuries. ${ }^{9}$ The condition is most frequently observed among individuals who participate in repetitive highimpact physical activities, like jumping and running. A previous epidemiological study estimated that the lifetime prevalence of Achilles tendinopathy among runners could be up to $52 \%$ among runners. ${ }^{10}$ It has been furtherly demonstrated that secondary career ending in these professional athletes was reported to be up to $5 \%$ in the affected personnel. $^{11}$ Another epidemiological investigation also assessed the prevalence of Achilles tendinopathy among 1400 included non-athlete participants. The authors reported that the prevalence of the condition was $5.6 \%$ among the included participants. Of these events, $3.6 \%$ were non-insertional while $4 \%$ were insertional, and $1.9 \%$ had combined lesions. ${ }^{12}$ Evidence indicates that the older population has a higher prevalence of chronic Achilles tendinopathy than the younger population. Furthermore, it has been shown that active individuals tend to have more insertional Achilles tendinopathy. On the other hand, Overweight, less active, and older individuals tend to have non-insertional Achilles tendinopathy more frequently than other populations. ${ }^{13}$ Furthermore, a previous study by Kvist et al reported that among athletes that had Achilles tendinopathy, around one-quarter of them were in young age groups. ${ }^{14}$

\section{Etiology}

Various observational studies have reported different causes of tendinopathy in sports. For instance, evidence shows that tendinopathy affecting the achilles tendon usually results from a chronic pathology following overuse of tendon among athletes. Different extrinsic and intrinsic factors have been associated with tendinopathy. These factors are adequately reported in the literature. The reported intrinsic factors include subtalar joint stiffness, inequal limb lengths, equinus, forefoot varus, and other biomechanical factors affecting the lower limbs, like cavus hindfoot deformity. ${ }^{15}$ Different systematic factors were also reported in the literature as major contributors to the development of Achilles tendinopathy. These factors include gout, obesity, hypertension, inflammatory arthropathies, diabetes, and old age. ${ }^{16-18}$ Other factors include the frequent administration of corticosteroids, quinolone antibiotics, aromatase inhibitors, and statin medications. Various extrinsic factors were also reported in the literature. ${ }^{19}$ These factors are mostly related to recurrent mechanical overload and excessive training errors.

Some of the reported factors include a rapid increase in mileage, training on sloping or hard surfaces, frequently changing training schedules, increased hill training, and excessive growth in interval training. Other reported factors include wedging from uneven wear and wearing running or footwear surfaces with modest shock absorption characteristics (particularly when changing from a normal position). ${ }^{15,20}$ Therefore, the etiology of developing Achilles tendinopathy results from the accumulation of these factors (leading to the weakened tendon) and 
repetitive microtrauma to the affected region. In addition, the reduced ability of the different body mechanisms to heal the impacted traumatic events affected the tendon also contributes to the condition's pathology and developing tendinopathy. Furthermore, evidence also shows that the pathology of Achilles tendinopathy might be elicited by poor blood flow to the central part of the tendon, increasing the risk and progression of the previously mentioned factors. $^{21,22}$

\section{Pathogenesis}

Mechanical overloading on the different tissues of tendons can significantly lead to various anabolic events. These events include increased synthesis of collagen proteins and upregulation of collagen gene expression. Previous studies have demonstrated that these favorable events usually peak at one day after overloading over the tendon and the complimentary events typically last for up to 70-80 hours. ${ }^{23,24}$ On the other hand, it has been demonstrated that other catabolic events also co-occur with the aforementioned anabolic effects of loading. These include degradation of collagen proteins, and evidence even shows that it might peak even before anabolic events. The net result of these events is the significant loss of collagen tissues within the first 24-36 hours. ${ }^{25}$ However, collagen gain occurs in the following hours. Therefore, it has been recommended that athletes should have adequate restitution time intervals to avoid the development and progression of adverse catabolic events. In addition, different anatomical factors related to the structure of tendons significantly contribute that the tendon has its specific functions of load endurance. These parameters include nerve-ending receptors, extracellular matrix, tenocytes, and tendon cells.

Microrupture of collagen fibers and increased inflammatory mediators production usually result from repetitive manipulation of tenocytes by overloading and strain events. Following repetitive mechanical overloading, it has been shown that the levels of inflammatory mediators are significantly increased. A crucial inflammatory mediator found in these events is Prostaglandin E2 (PGE2). ${ }^{26}$ Previous studies reported that a histological pattern of tendinopathy might result from the peritendinous administration of PGE1. In the same context, it has been shown that significant degenerative changes were observed following the administration of intratendinous PGE2. ${ }^{27,28}$ Thus, current evidence indicates that inflammatory changes play an essential part in the pathogenesis of tendinopathy. This is in addition to different granulomatous effects on the surrounding blood vessels.

Studies show that the affected regions have abundant infiltration by $\mathrm{T}$ and $\mathrm{B}$ lymphocytes, mast cells, and macrophages. ${ }^{29,30}$ These findings also show the essential role of intrinsic immune functions in mediating the pathogenesis of tendinopathy. As a result of these events, activation of the inflammatory cascade following the mentioned cells usually develops. Examples of the inflammatory mediators include interleukin (IL)-6, IL-15, and IL-18. ${ }^{31}$ It has been furtherly shown that fibroblasts and tendon cells can remarkably turn into myofibroblasts. This occurs secondary to being stimulated by transforming growth factor $\beta$ (TGF- $\beta$ ) and inflammatory cytokines, in addition to being subjected to repetitive traumatic events.

The biological importance of the presence of myofibroblasts includes tissue healing and adaptation. ${ }^{32}$ However, it has been indicated that the fat of these myofibroblasts is apoptosis. ${ }^{32}$ This occurs after completing their functions of tissue healing and adaption following the release of mechanical stressors. However, in cases when this process fails, the formulated myofibroblasts will undergo fibrosis and hyperproliferation. This has been observed as a main histological feature in tendinopathy. Furthermore, hypoxia can also contribute to the hyperproliferation of fibroblasts. ${ }^{33}$ This has been associated with altered tendon-related material composition secondary to upregulation of the matrix metalloproteinases. ${ }^{34}$ It has been furtherly reported that vascular endothelial growth factor is usually upregulated following hypoxia. This contributes to tendinopathy due to the potential increase in the process of angiogenesis. ${ }^{34} \mathrm{~A}$ previous study also suggested that associated pain with tendinopathy results from angiogenesis. This is logical because diminished pain parameters are usually associated with sclerotherapy. ${ }^{35}$ This is attributed to the growth of nerve fibers together with the newly formed blood vessels.

On the other hand, the per se development of blood vessels is not a painful process. ${ }^{30,36,37}$ This can be indicated by the fact that healthy tendons are nonpainful, non-neuronal in the standard physiological settings. On the other hand, increased growth of nerve fibers and release of a nociceptive substance that leads to significant painful sensations are usually noticed in cases of chronicallyinflamed tendons. ${ }^{36,37}$ Relieving pain and reducing its severity can be done by denervation and resectioning the growing pathological nerve fibers. ${ }^{38}$ This can be achieved by releasing the paratenon or using a minimally-invasive surgical procedure. Ingrowth of these nerve fibers and the development of painful events have been reported secondary to traumatic injuries and repetitive loading. ${ }^{39}$

However, such events are not usually observed during standard settings. This is because the ingrowth of nerve fibers and subsequent release of a nociceptive substance is usually encountered by the ingrowth of corresponding autonomic nerve fibers. This significantly leads to nerve retraction and reduced nociception. ${ }^{3,40}$ No retraction occurs in cases of tendinopathy. Such processes contribute to the pathogenesis of the condition by reduced healing of wounds secondary to the aberrant sprouting of sensory nerves and neuronal dysregulation.

Accordingly, in cases of tendinopathy, evidence indicates increased hyperproliferative changes and increased pain signaling. ${ }^{37}$ Different functions were also reported 
secondary to the development of these peripheral nerve fibers. It has been shown that these fibers release abundant amounts of chemical substances in response to mechanical stimulation.

These substances are suitable for hemostasis and healing. However, prolonged-release can cause fibrosis. ${ }^{36}$ Old and recent investigations have adequately documented the presence of nerve receptors and mediators in tendons. ${ }^{36,41}$ Increased levels of substance $\mathrm{P}$ and neuropeptides were observed during tendinopathy. These factors contribute to the development of atrophic changes and inflammation, and the discussed nociceptive functions.

Mechanical loading can upregulate the release of substance P. ${ }^{30,36,37}$ The latter has been associated with neurokinin 1, cytokines, plasma extravasation, and vasodilatation. It has been further shown that substance $\mathrm{P}$ has been associated with increased levels of TGF- $\beta$, leading to the transformation of fibroblasts into myofibroblasts. ${ }^{36,42-46} \mathrm{It}$ also has a potential function over endothelial proliferation. Therefore, abnormal regulation of substance $\mathrm{P}$ can be associated with hypervascularization, hypercellularity, tenocyte transformation, and fibrosis.

These pathological changes are well-demonstrated among patients with tendinopathy. Increased upregulation of the neurotransmitter glutamate was also evidenced in these events. ${ }^{47-49}$ Evidence shows that this substance plays an essential role in the pathology of different painful conditions. Recent evidence furtherly confirms its role in the pathogenesis of tendinopathy. ${ }^{3}$ Figure 1 demonstrates the morphological changes observed in tendinopathy compared to normal tendon structure.
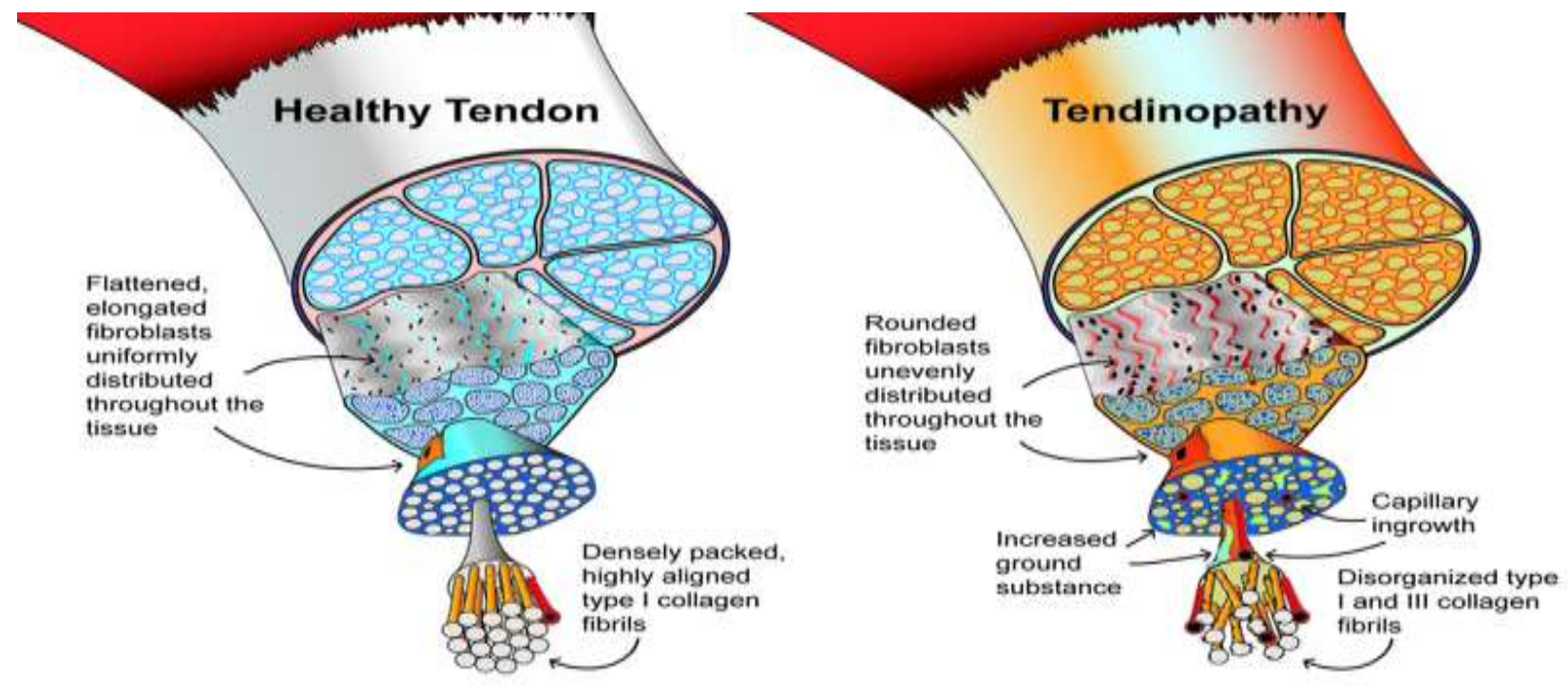

Figure 1: Morphological changes of tendinopathy compared to normal tendon. ${ }^{50}$

\section{CONCLUSION}

Although tendinopathy might seem a non-critical condition, estimates show that it might be career-ending for many professional athletes, and therefore, reducing their quality of life. In the present literature review, we have discussed the epidemiology, etiology, and pathogenesis of tendinopathy among athletes. Different intrinsic and extrinsic factors were found to contribute to the development of the condition and the impact of various systemic diseases. Evidence also shows that the prevalence of the condition is high among athletes. However, these rates were variable across the different studies in the literature. This is probably owing to various factors, like demographics and the process of detection. The pathogenesis of the condition is also very complicated, and the development of pain has been attributed to the process of angiogenesis and associated ingrowth of nerve fibers.

Funding: No funding sources Conflict of interest: None declared Ethical approval: Not required

\section{REFERENCES}

1. Woo SL, Renström PA, Arnoczky SP. Tendinopathy in athletes. Hong Kong: John Wiley and Sons; 2008.

2. Khan KM, Maffulli N. Tendinopathy: an Achilles' heel for athletes and clinicians. Clin J Sport Med. 1998;8(3):151-4.

3. Ackermann PW, Renström P. Tendinopathy in sport. Sports Health. 2012;4(3):193-201.

4. Sharma P, Maffulli N. Tendon injury and tendinopathy: healing and repair. J Bone Joint Surg Am. 2005;87(1):187-202.

5. Mead MP, Gumucio JP, Awan TM, Mendias CL, Sugg KB. Pathogenesis and Management of Tendinopathies in Sports Medicine. Transl Sports Med. 2018;1(1):5-13.

6. Kujala UM, Sarna S, Kaprio J. Cumulative incidence of achilles tendon rupture and tendinopathy in male former elite athletes. Clin J Sport Med. 2005;15(3):133-5. 
7. Hägglund M, Zwerver J, Ekstrand J. Epidemiology of patellar tendinopathy in elite male soccer players. Am J Sports Med. 2011;39(9):1906-11.

8. Cook JL, Purdam CR. Is tendon pathology a continuum? A pathology model to explain the clinical presentation of load-induced tendinopathy. $\mathrm{Br} \mathrm{J}$ Sports Med. 2009;43(6):409-16.

9. Sobhani S, Dekker R, Postema K, Dijkstra PU. Epidemiology of ankle and foot overuse injuries in sports: A systematic review. Scand J Med Sci Sports. 2013;23(6):669-86.

10. Maffulli N, Wong J, Almekinders LC. Types and epidemiology of tendinopathy. Clin Sports Med. 2003;22(4):675-92.

11. Lysholm J, Wiklander J. Injuries in runners. Am J Sports Med. 1987;15(2):168-71.

12. Waldecker U, Hofmann G, Drewitz S. Epidemiologic investigation of 1394 feet: coincidence of hindfoot malalignment and Achilles tendon disorders. Foot Ankle Surg. 2012;18(2):119-23.

13. Li HY, Hua YH. Achilles Tendinopathy: Current Concepts about the Basic Science and Clinical Treatments. Biomed Res Int. 2016;2016:6492597.

14. Kvist M. Achilles tendon injuries in athletes. Ann Chir Gynaecol. 1991;80(2):188-201.

15. Irwin TA. Current concepts review: insertional achilles tendinopathy. Foot Ankle Int. 2010;31(10):933-9.

16. Tuite DJ, Renström PA, O'Brien M. The aging tendon. Scand J Med Sci Sports. 1997;7(2):72-7.

17. Holmes GB, Lin J. Etiologic factors associated with symptomatic achilles tendinopathy. Foot Ankle Int. 2006;27(11):952-9.

18. Paavola M, Kannus P, Järvinen TA, Khan K, Józsa L, Järvinen M. Achilles tendinopathy. J Bone Joint Surg Am. 2002;84(11):2062-76.

19. Knobloch K. Drug-Induced Tendon Disorders. Adv Exp Med Biol. 2016;920:229-38.

20. Kirchgesner T, Larbi A, Omoumi P, Malghem J, Zamali N, Manelfe J, Lecouvet F, et al. Drug-induced tendinopathy: from physiology to clinical applications. Joint Bone Spine. 2014;81(6):485-92.

21. Son PT, Reda A, Viet DC. Exchange transfusion in the management of critical pertussis in young infants: a case series. Vox Sang. 2021;116(9):976-82.

22. Qushayri AE, Dahy A, Reda A, Mahmoud MA, Mageed SA, Kamel AMA, et al. A closer look at the high burden of psychiatric disorders among healthcare workers in Egypt during the COVID-19 pandemic. Epidemiol Health. 2021;43:2021045.

23. Miller BF, Olesen JL, Hansen M, Døssing S, Crameri RM, Welling RJ, et al. Coordinated collagen and muscle protein synthesis in human patella tendon and quadriceps muscle after exercise. J Physiol. 2005;567(3):1021-33.

24. Heinemeier KM, Olesen JL, Haddad F, Langberg H, Kjaer M, Baldwin KM, Schjerling P. Expression of collagen and related growth factors in rat tendon and skeletal muscle in response to specific contraction types. J Physiol. 2007;582(3):1303-16.
25. Magnusson SP, Langberg $H$, Kjaer $M$. The pathogenesis of tendinopathy: balancing the response to loading. Nat Rev Rheumatol. 2010;6(5):262-8.

26. Wang JH, Jia F, Yang G, Yang S, Campbell BH, Stone D, Woo SL. Cyclic mechanical stretching of human tendon fibroblasts increases the production of prostaglandin E2 and levels of cyclooxygenase expression: a novel in vitro model study. Connect Tissue Res. 2003;44(3):128-33.

27. Khan MH, Li Z, Wang JH. Repeated exposure of tendon to prostaglandin-E2 leads to localized tendon degeneration. Clin J Sport Med. 2005;15(1):27-33.

28. Sullo A, Maffulli N, Capasso G, Testa V. The effects of prolonged peritendinous administration of PGE1 to the rat Achilles tendon: a possible animal model of chronic Achilles tendinopathy. J Orthop Sci. 2001;6(4):349-57.

29. Millar NL, Hueber AJ, Reilly JH, Xu Y, Fazzi UG, Murrell GA, McInnes IB. Inflammation is present in early human tendinopathy. Am J Sports Med. 2010;38(10):2085-91.

30. Schubert TE, Weidler C, Lerch K, Hofstädter F, Straub RH. Achilles tendinosis is associated with sprouting of substance $\mathrm{P}$ positive nerve fibres. Ann Rheum Dis. 2005;64(7):1083-6.

31. Millar NL, Wei AQ, Molloy TJ, Bonar F, Murrell GA. Cytokines and apoptosis in supraspinatus tendinopathy. J Bone Joint Surg Br. 2009;91(3):41724.

32. Tomasek JJ, Gabbiani G, Hinz B, Chaponnier C, Brown RA. Myofibroblasts and mechano-regulation of connective tissue remodelling. Nat Rev Mol Cell Biol. 2002;3(5):349-63.

33. Freeman TA, Parvizi J, Dela Valle CJ, Steinbeck MJ. Mast cells and hypoxia drive tissue metaplasia and heterotopic ossification in idiopathic arthrofibrosis after total knee arthroplasty. Fibrogenesis Tissue Repair. 2010;3:17.

34. Pufe T, Petersen WJ, Mentlein R, Tillmann BN. The role of vasculature and angiogenesis for the pathogenesis of degenerative tendons disease. Scand J Med Sci Sports. 2005;15(4):211-22.

35. Ohberg L, Alfredson H. Ultrasound guided sclerosis of neovessels in painful chronic Achilles tendinosis: pilot study of a new treatment. Br J Sports Med. 2002;36(3):173-5.

36. Ackermann PW, Li J, Lundeberg T, Kreicbergs A. Neuronal plasticity in relation to nociception and healing of rat achilles tendon. J Orthop Res. 2003;21(3):432-41.

37. Lian $\varnothing$, Dahl J, Ackermann PW, Frihagen F, Engebretsen L, Bahr R. Pronociceptive and antinociceptive neuromediators in patellar tendinopathy. Am J Sports Med. 2006;34(11):18018.

38. Sterkenburg MN, van Dijk CN. Mid-portion Achilles tendinopathy: why painful? An evidence-based philosophy. Knee Surg Sports Traumatol Arthrosc. 2011;19(8):1367-75. 
39. Messner K, Wei Y, Andersson B, Gillquist J, Räsänen T. Rat model of Achilles tendon disorder. A pilot study. Cells Tissues Organs. 1999;165(1):30-9.

40. Ackermann PW, Salo PT, Hart DA. Neuronal pathways in tendon healing. Front Biosci (Landmark Ed). 2009; 14:5165-87.

41. Scott A, Bahr R. Neuropeptides in tendinopathy. Front Biosci (Landmark Ed). 2009;14:2203-11.

42. Schizas N, Li J, Andersson T, Fahlgren A, Aspenberg $\mathrm{P}$, Ahmed M, et al. Compression therapy promotes proliferative repair during rat Achilles tendon immobilization. J Orthop Res. 2010;28(7):852-8.

43. Ljung BO, Alfredson H, Forsgren S. Neurokinin 1receptors and sensory neuropeptides in tendon insertions at the medial and lateral epicondyles of the humerus. Studies on tennis elbow and medial epicondylalgia. J Orthop Res. 2004;22(2):321-7.

44. Hoffmann P, Hoeck K, Deters S, Martini I, Schmidt WE. Substance $P$ and calcitonin gene related peptide induce TGF-alpha expression in epithelial cells via mast cells and fibroblasts. Regul Pept. 2010;161(1):33-7.

45. Bring DK, Reno C, Renstrom P, Salo P, Hart DA, Ackermann PW. Joint immobilization reduces the expression of sensory neuropeptide receptors and impairs healing after tendon rupture in a rat model. J Orthop Res. 2009;27(2):274-80.

46. Andersson G, Danielson P, Alfredson H, Forsgren S. Presence of substance $\mathrm{P}$ and the neurokinin-1 receptor in tenocytes of the human Achilles tendon. Regul Pept. 2008;150(1):81-7.

47. Schizas N, Lian $\varnothing$, Frihagen F, Engebretsen L, Bahr R, Ackermann PW. Coexistence of up-regulated NMDA receptor 1 and glutamate on nerves, vessels and transformed tenocytes in tendinopathy. Scand J Med Sci Sports. 2010;20(2):208-15.

48. Molloy TJ, Kemp MW, Wang Y, Murrell GA. Microarray analysis of the tendinopathic rat supraspinatus tendon: glutamate signaling and its potential role in tendon degeneration. J Applied Physiol. 2006;101(6):1702-9.

49. Alfredson H, Forsgren S, Thorsen K, Fahlström M, Johansson H, Lorentzon R. Glutamate NMDAR1 receptors localised to nerves in human Achilles tendons. Implications for treatment? Knee Surg Sports Traumatol Arthrosc. 2001;9(2):123-6.

50. Alfredson H, Forsgren S, Thorsen K, Fahlström M, Johansson H, Lorentzon R. Glutamate NMDAR1 receptors localised to nerves in human Achilles tendons. Implications for treatment? Knee Surg Sports Traumatol Arthrosc. 2001;9(2):123-6.

Cite this article as: Hasan SA, Othman AA, Shagdar YK, Khan AA, Sulaimani AB, Alsinan HA, et al. Epidemiology, etiology, and pathogenesis of tendinopathy among athletes. Int J Community Med Public Health 2022;9:437-42. 